\title{
COMMENTARY
}

\section{Engaging In Authentic Allyship As Part of Our Professional Development} \author{
Nancy Borja-Hart, PharmD, ${ }^{\mathrm{e}}$ Latasha Wade, PharmD, ${ }^{\mathrm{f}}$ Brianna Henson, MPA ${ }^{\mathrm{g}}$ \\ ${ }^{\text {a }}$ Midwestern University, College of Pharmacy-Downers Grove, Downers Grove, Illinois \\ ${ }^{\mathrm{b}}$ Midwestern University, College of Pharmacy-Glendale, Glendale, Arizona \\ ${ }^{\mathrm{c}}$ University of Michigan, College of Pharmacy, Ann Arbor, Michigan \\ ${ }^{\mathrm{d}}$ University of the Sciences in Philadelphia, College of Pharmacy, Philadelphia, Pennsylvania \\ ${ }^{\mathrm{e}}$ University of Tennessee Health Science Center, College of Pharmacy, Memphis, Tennessee \\ ${ }^{\mathrm{f}}$ American Association of Colleges of Pharmacy, Arlington, Virginia \\ ${ }^{\mathrm{g}}$ University of Kentucky, College of Pharmacy, Lexington, Kentucky
}

Sally Arif, PharmD, ${ }^{\mathrm{a}}$ Titilola Afolabi, PharmD, ${ }^{\mathrm{b}}$ Beatriz Manzor Mitrzyk, PharmD, ${ }^{\mathrm{c}}$ Tyan F. Thomas, PharmD, MSMEd,

Corresponding Author: Sally Arif, Midwestern University, College of Pharmacy, 555 31st Street, Downers Grove, IL 60515. Tel: 630-515-6466. Email: sarif@ midwestern.edu

Submitted April 14, 2021; accepted August 3, 2021; ePublished August 2021

Educational institutions increasingly recognize the importance of diversity, equity and inclusion (DEI) efforts to combat and dismantle structures that sustain inequities. However, successful DEI work hinges on individuals being authentic allies and incorporating allyship into their professional development. Allyship involves members of dominant groups recognizing their privilege and engaging in actions to create inclusivity and equitable spaces for all. Often times, individuals from dominant groups with desires to actively support others from marginalized groups are unsure how to fight oppression and prejudice. Our goal, as faculty with diverse perspectives and heterogeneous intersectional identities, is to provide readers with the tools to develop as an authentic ally through: 1) educating themselves about the identities and experiences of others, 2) challenging their own discomfort and prejudices, 3) dedicating the time and patience to learning how to be an ally, and 4) taking action to promote change toward personal, institutional, and societal justice and equality. Ultimately, each person must advocate for change because we all hold the responsibility. When everyone is an authentic ally, we all thrive and rise together.

Keywords: diversity, inclusion, equity, allyship, cultural taxation

Recent national events including the death of George Floyd and the COVID-19 pandemic have highlighted the reverberating impact of inequities within our society and the vital importance of purposeful and intentional diversity, equity and inclusion (DEI) work. Educational institutions, the bedrock of fomenting knowledge in our society, are also scouring for ways to better support colleagues and students from marginalized backgrounds. National organizations and academic institutions are stating their positions, and many recognize the need for DEI advocates in leadership positions. Pharmacy academia is not exempt from this quest and on an individual level, faculty and staff are asking themselves "What more can I do?" Despite one's belief that they are "a good person," many individuals continue to ignore inequities. They might be paralyzed by discomfort, afraid to cause friction, or lack awareness and knowledge about social justice actions. Either way, we are responsible for the inequity if we do nothing to change it. To create safer, more respectful, and equitable campus communities and accomplish our institutional DEI goals, we must recognize and emphasize that the responsibility for creating change falls on every individual. Faculty within the pharmacy profession must rise to the task, deeply reflect, embrace discomfort, and take action to dismantle the structures that sustain inequities. One way to intentionally contribute to such change on an individual basis is by embracing authentic allyship as part of our professional development.

An ally is an individual from a dominant group who seeks to end oppression by actively engaging in improving the lives of marginalized individuals through intentional and conscious efforts. ${ }^{1}$ Allies transfer the benefits of their privilege to those who lack access to this privilege. An authentic ally educates themselves, promotes DEI through their actions, addresses their implicit biases, and provides support to those in need, while embracing the complexity and discomfort of DEI work. Being an ally starts with self-reflection by asking: What am I doing to change the structural barriers present in the pharmacy academy? What am I doing daily to help create meaningful change towards equity in my spheres of influence?

Most individuals in the dominant culture were taught that hard work and desire for success are how to achieve your goals. ${ }^{2}$ Despite the same amount of hard work and dedication by many from marginalized groups, success is often times more difficult to achieve due to barriers imposed by systems of oppression. Institutions of higher education are not immune 
to the systemic barriers that disadvantage marginalized groups. Cultural taxation, or the extra work marginalized faculty endure carrying out ethnic representation for the university, can limit equitable achievement or career advancement since the additional responsibilities are often unrewarded. ${ }^{3}$ For example, faculty of color may serve for several years on the same committees because of a need for representation, limiting their time to assume other roles or pursue other professional advancements. This form of cultural taxation can further exacerbate disparities as seen with leadership roles or distinguished positions often being assumed by privileged identities. In the U.S., privileged identities include U.S. citizens, male gender, White race (ie, European ancestry, settler), affluent/financially secure, heterosexual, cis-gendered, thin, able-bodied, and Christian. Marginalization of people often occurs based on race, gender, sexual orientation, national origin, English language proficiency, just to name a few. ${ }^{4,5}$

An authentic ally challenges the status quo that permits inequities. They do not treat allyship as an identity or a badge of honor, but rather a commitment to a lifelong journey that requires constant self-critique and the practice of cultural humility. Authentic allyship should not be fueled by self-interest because one sees themself as a savior or protector of those who experience oppression as this is characteristic of performative allyship. ${ }^{6}$ Authentic allyship is not self-defined but rather recognized by marginalized groups.

Being an ally does not necessarily mean you fully understand what it feels like to be oppressed. It means you are willing to take on the struggle as your own and "walk the walk" not just "talk the talk." It requires pushing beyond shame or guilt around having privilege in order to move the needle towards a more equitable world. It also requires one to acknowledge that even if you feel upset, redirecting your attention and actions to dismantling inequities is more important than self-discomfort. While there is no one-size-fits all approach for allyship, it is everyone's responsibility to start somewhere. Here are some points to consider as you approach allyship authentically in your classroom, practice setting, campus, and community (refer to Table 1 for situational examples and reflective steps):

\section{Expect to make (a lot of) mistakes.}

Mistakes will happen and we must own them, not use them as a justification for inaction. This makes educating ourselves about the identities and experiences of others even more vital. Being open to honest feedback is an incredibly important skill to develop as an ally. It may be easier, emotionally, to be corrected about something we do not know if we have a growth mindset and embrace the opportunity of learning. On the other hand, we cannot always assume we will be corrected, and it will not always be clear that we have made a mistake.

\section{Seek to learn more and engage with others}

A true ally will acknowledge the limits of their knowledge regarding the experiences of marginalized groups and seek opportunities to learn and grow. An ally should exercise empathy, which requires one to understand what it is like to walk in another person's shoes. Empathy drives forward action and requires us to have a deeper understanding of one another. Take time to learn about your colleagues beyond their workplace aspirations if they are open to such exploration. Learn about the language and identities of your marginalized colleagues and students. Dedicate time to learn how to pronounce their names, what they call their disabilities, their ethnicity, their religion, their preferred pronouns, and interests. Approach conversations from a place of cultural curiosity instead of relying on stereotypes or making assumptions. It is okay to ask questions about another person's experiences. However, if the answers can be found in accessible resources such as books and podcasts, you are adding unnecessarily burden on the individual and not being an authentic ally. Finally, be patient with your inquiries and give time for people to open up and build trust. People from traditionally marginalized communities often face microaggressions, so it may take time for them to ascertain if your inquiries are rooted in genuine curiosity or are being posed to affirm harmful stereotypes.

\section{Listen more, talk less}

Pass the mic, do not interrupt, and actively listen. To be an authentic ally, actively listen when engaging students after class, have brief workplace hallway chats, or attend faculty meetings. For example, during faculty or committee meetings, pay attention to how much time you contribute to the discussion compared to your colleagues from marginalized groups. If you are a regular contributor to discussions at these meetings, relinquish some of your air-time so that others can contribute and be heard.

\section{Say something, even if it is uncomfortable}

Allies stand up for others when they are witness to overt or covert discrimination, harassment, or microaggressions. We must understand what microaggressions are, avoid making them, and call them out when they happen around us. When we ignore these negative behaviors, we deflect responsibility and perpetuate the problem. In pharmacy practice, we may encounter interactions with patients or other health care providers that put our students from marginalized populations in 
uncomfortable situations because of being perceived as different. If your student shares that they have experienced discrimination, harassment, or microaggressions, listen to your student and ask them what they think would be a good solution or offer to problem-solve together. An authentic ally advocates for students and encourages others to do so as well.

\section{Amplify suppressed voices before your own}

Echo the ideas shared by marginalized colleagues and students, and give them credit for their ideas. We cannot speak on behalf of marginalized individuals, but we can assure they are heard and recognized for their ideas. Amplify the voices of your marginalized colleagues and students with a genuine desire to support and promote equity, not from a need to "rescue" marginalized groups or appear progressive. For example, the chair of the curriculum committee is asked by students who identify as transgender to include transgender health to the curriculum. Being an authentic ally, the chair sees this as an opportunity and invites these students to present their ideas and recommendations to the curriculum committee. Another example is a course director offering additional support to students preparing for experiential rotations by asking the students to express their needs and concerns. Authentic allies allow voices from marginalized groups to be heard and encourage others to do the same.

\section{Become a professional sponsor}

Authentic allies create space at the podium for those of marginalized groups. Professional sponsorship goes beyond mentorship, which is traditionally seen as an individual who supports junior colleagues in shaping their professional development. Sponsors are individuals with significant influence in institutional decision-making, who advocate and fight for career advancement of others, investing in platforms to promote others at the risk of their own reputations. ${ }^{7}$ Sponsors are keenly aware of their own privilege and purposefully disseminate benefits of such privileges to lift marginalized groups. Professional sponsors in academia must consciously steer away from the natural inclination to provide sponsorship to individuals based on a sense of kinship or commonality (eg, "they remind me of myself" attitude). Such inclinations enrich individuals with whom the sponsor shares mutual characteristics, consequently reducing or not making available opportunities for individuals who belong to marginalized groups. Professional sponsors are often not sought after or offered to mentees but may develop as a strategic alliance initiated by a sponsor to promote diversity in higher leadership networks.

\section{Do more than be an ally, become an advocate}

Becoming an ally is an important first step. Assuming a more active role means becoming an advocate. It involves readily supporting and promoting a marginalized student, faculty member, or patient when they say they are experiencing microaggressions or do not feel included. More pharmacy educators and pharmacists are needed to take on the role of advocate because structural racism is easier to ignore if only the marginalized protest it.

While a small percentage of faculty and staff identify as members of marginalized groups, these individuals often disproportionately bear the burden of articulating, implementing, and carrying out institutional DEI goals. ${ }^{8}$ Given the relatively large proportion of the academy that identifies as a member of the dominant culture, allyship must be cultivated at all levels within our institutions to amplify the message and to spread the load of DEI work. Individuals with privilege must make a personal commitment to become an ally by continuously: 1) educating themselves about the identities and experiences of others, 2) challenging their own discomfort and prejudices, 3) dedicating the time and patience to learning how to be an ally, and 4) taking action to promote change toward personal, institutional, and societal justice and equality. ${ }^{6}$ Taking small and consistent steps is better than large inconsistent leaps. Find and leverage your capacity and start somewhere, even if this is about starting with learning more about an ally (table 2 for further resources and readings). This is not about "arriving" at a DEI finish line, but rather engaging in a lifelong learning process. We applaud the efforts made by faculty, staff, students, and institutions within the Academy, and acknowledge there is still work to be done. When each individual is an authentic ally, we all thrive and rise together.

\section{REFERENCES:}

1. Bishop A. Becoming an ally: Breaking the cycle of oppression in people. 2nd ed. Halifax, Nova Scotia: Fernwood Publishing; 2002. [Google Scholar]

2. Boucher B \& Chisholm-Burns. Black Lives Do Matter. AJPE. 2020; 84 (11): 8306. [Google Scholar]

3. Padilla A.M. Ethnic Minority Scholars, Research, and Mentoring: Current and Future Issues. Educational Researcher. 1994; 23(4): 24-27. [Google Scholar]

4. Monohan M.J. The concept of privilege: A critical appraisal. South African Journal of Philosophy. 2014; 33(1): 73-83. [Google Scholar]

5. Collins CS, Jun, A. White out: Understanding White privilege and dominance in a modern age. New York, NY: Peter Lang; 2017. 
6. Edwards KE. Aspiring Social Justice Ally Identity Development: A Conceptual Model. NASPA Journal. 2006;43 (4): 39-60. [Google Scholar]

7. Raphael J. The role of sponsorship in achieving workforce diversity in academic pediatrics. Pediatrics; Aug 2019; 144 (2) e20191532. [ Google Scholar]

8. National Center for Education Statistics, U.S. Department of Education. The Condition of Education 2017 (NCES 2017-144). https://nces.ed.gov/fastfacts/display.asp?id=61. Accessed April 8, 2021.

9. Office of the Provost, Columbia University. Guide to Best Practices in Faculty Mentoring. https://provost.columbia.edu/sites/default/files/content/Best\%20Practices\%20in\%20Faculty\%20Mentoring.pdf Accessed 16 June 2021. 
Table 1. Characteristics of an Authentic Ally and Situational Examples

\section{Characteristics of An $\quad$ Situational Examples Authentic Ally}

\begin{tabular}{ll}
\hline $\begin{array}{l}\text { Expect to make ( } \text { ( lot of) } \\
\text { mistakes. }\end{array}$ & Consider asking "How did you feel when I ...?" after professional interactions with colleagues or \\
& students to gain perspective. \\
& Considering incorporating questions like "Was there a better way I could have ...?" in post-course \\
& anonymous student-evaluations and class activities.
\end{tabular}

Seek to learn more and engage with others

Listen more, talk less

Say something, even if it is uncomfortable

Amplify suppressed voices before your own

Become a professional sponsor, or expand your sponsorship to a more diverse group

Do more than be an ally, become an advocate
Start by reading DEI books, watch YouTube videos and TED talks, and follow DEI topics on social media or just ask your DEI leaders for resources to broaden your understanding.

As faculty, we can mentor, sponsor, and give opportunities to marginalized students in our programs and be an authentic ally by asking students how they would like our support.

Working with faculty committees and student groups that support marginalized populations is another way to gain understanding, connection, and learn to be a better ally.

Consider reflecting on the following after attending a committee meeting:

Did I dominate the conversation?

Did I get credit for ideas that were not your own, or did I give credit to the individual with the original idea?

Did I ignore the ideas of others, or did I acknowledge differences of thought and respectfully disagree? At a future meeting if you disagree with or do not understand your colleague's position, you can start by simply stating your position, such as "As a [insert your identity], I have never had your experience, but I'd really like to hear how you feel about ...."

One of your patients may overhear your student on experiential rotation speaking with another patient in their native language and share with you that it is disrespectful to speak a language other than English in the U.S. Be willing to stand up for your student.

If you find yourself on a committee discussing the needs of a particular group and you look around and don't see anyone who identifies as a member of that group in the conversation, question any conclusions that were reached.

Questions that could be included on faculty surveys by Chairs or Administrators could include "How can I best help support you?" and "What do I need to know to best help you succeed?" An ally demonstrates support through actions.

Give students an opportunity to discuss concerns or ideas during class time and ask other students to listen with an open mind by clearly stating these expectations at the beginning of class time and in your syllabus as part of the "rules of engagement."

Sponsors often initiate sponsorship of a more junior individual with whom they identify. ${ }^{9}$ Ask yourself the following questions about who you choose to sponsor: Who have I sponsored in the past? What prompted me to sponsor these individuals? Do I share similar characteristics to those who I have sponsored in the past? (eg, similar upbringing, culture, religious beliefs, family status, work style) Have I sponsored individuals who are different from me? Have I considered sponsoring someone who is different from me, and if so, why did I pursue or not pursue sponsorship?

A colleague confides that they volunteered to lead a committee but the request has never been acknowledged. You can speak to others in the committee and provide evidence as to why your colleague deserves the position.

A patient tells you that they have a medical complaint that they have voiced to other health care providers and feel it is being ignored. You can document the patient's complaint in your note and state that you are alerting the health care provider. If appropriate, you could recommend possible treatment if it is in the scope of your clinical practice.

A female student tells you they feel uncomfortable demonstrating blood pressure monitoring skills on a male student based on cultural beliefs. Ask the student if there are other partners they would feel comfortable practicing with and facilitate this change.

Acknowledge and use your privilege to participate in these types of controversial situations on behalf of students, faculty, and patients who cannot afford to because the repercussions for them are so much greater or because no one else will listen. 
Guide to allyship

https:/guidetoallyship.com/

Anti-racism and allyship 7 day journey

https://gsb-sites.stanford.edu/anti-racism-and-allyship/

The anti-oppression network.

https://theantioppressionnetwork.com/allyship/

Rochester racial justice toolkit

https://thetoolkit.wixsite.com/toolkit/guide

The Pharmacists' Defence Association \& The PDA Union https://www.the-pda.org/get-involved/networks/bame/allyship/

Navigating Allyship through Indigenization, Decolonization, and Reconciliation: Perspectives from Non-Indigenous Pharmacy Educators

https://pubs.lib.umn.edu/index.php/innovations/article/view/2300/2344

The dismantle collective

https://www.dismantlecollective.org/resources/

Ohio Northern University. Heterick Library

https://library.onu.edu/c.php?g=1049073\&p=7616446

George Washington Office for Diversity, Equity, and Community Engagement:Resources on Allyship https://diversity.gwu.edu/resources-allyship
An open source starter guide to help you become a more thoughtful and effective ally.

You want to begin your journey to learn more about antiracism and allyship.

Compiled information from multiple websites about allyship.

Toolkit for individuals based on their perspective, identity, or role.

Reading suggestions to support allyship specific to pharmacists.

Practical applications of allyship through the lens of indigenization, decolonization, and reconciliation.

White Allyship 101: Resources to Get to Work

Anti-Racism Resources including links to videos and TED talks

Links to various allyship websites and recommendations on books on allyship 